\title{
Multilepton production at HERA
}

UH Monica Turcato

iti Hamburg University

SPONSORED BY THE

Federal Ministry
of Education

and Research

zEUs on behalf of the ZEUS and H1 Collaborations

XVII International Workshop on Deep Inelastic

Scattering and Related Subjects

Madrid, Spain

April 26-30, 2009 


\section{HERA physics}

HERA: $\mathrm{e}^{ \pm} \mathrm{p}$ collider, $\mathrm{E}_{\mathrm{cm}} \sim 318 \mathrm{GeV}$

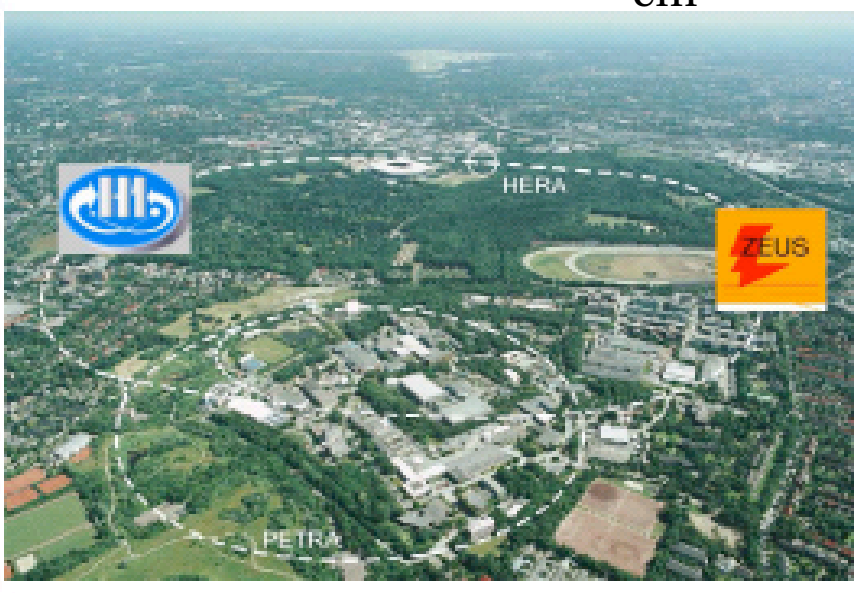

ZEUS and H1: multipurpose experiments located in two of the interaction points.
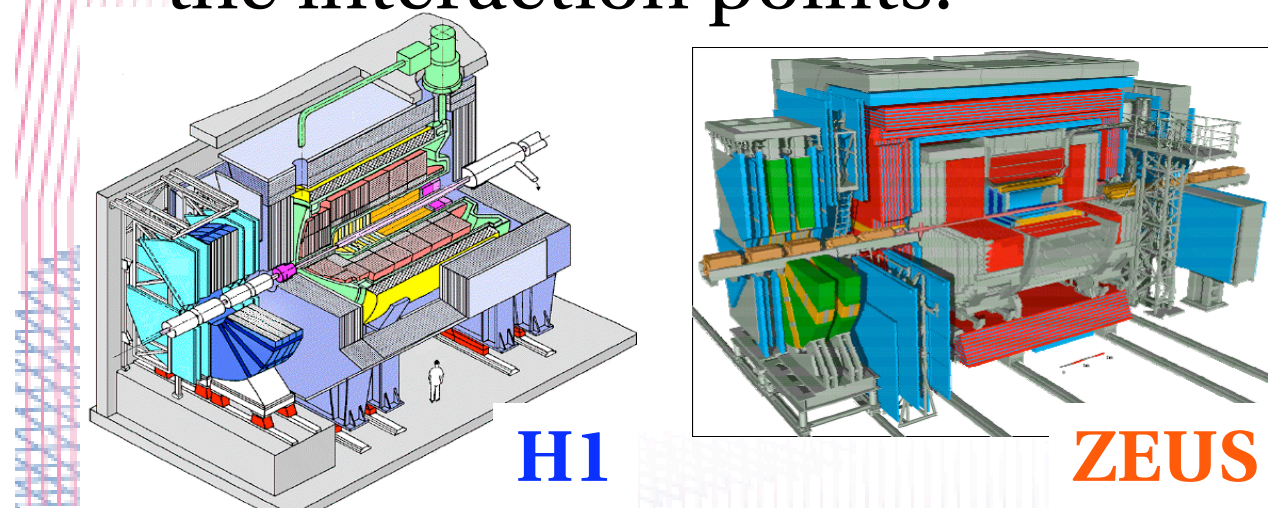

U+
Data taking ended in June 07.

Collected luminosity :

$\sim 0.5 \mathrm{fb}^{-1}$ per experiment

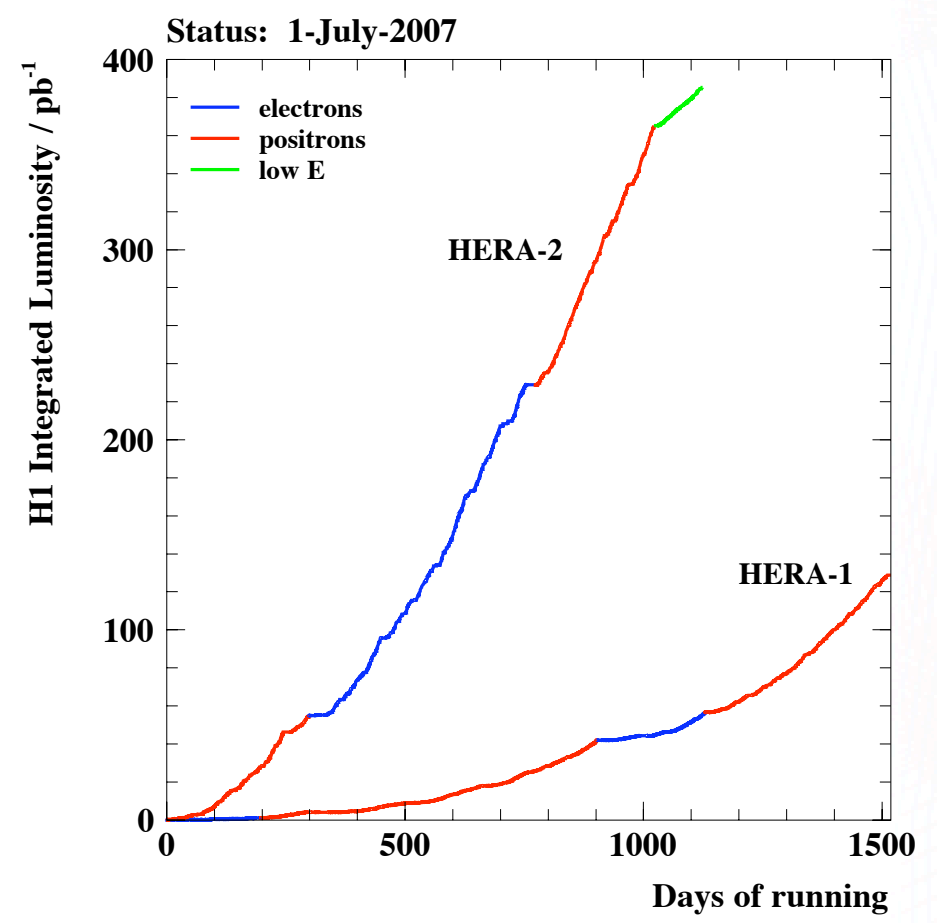

Rare ( $\sigma \sim 1 \mathrm{fb})$ phenomena may become visible. 


\section{Outline}

- Events in which two or more isolated electrons or muons with high transverse momentum are found give a clean experimental signature where to look for signatures of beyond the Standard Model (SM) physics.

- The final results of the single H1 and ZEUS analyses will be shown.

- The combination of the data of the two experiments allows a more stringent test of the SM in the interesting phase space regions: preliminary results on a common phase space based on $0.94 \mathrm{fb}^{-1}$ will be shown.

- Di- $\tau$ production with decay into leptons is not vetoed in the analysis. Hadronic $\tau$ production is removed by the cuts. Preliminary results on $\tau$ production from ZEUS exist but are not shown here (focus on high- $\mathrm{p}_{\mathrm{T}}$ multi-leptons). 


\section{Multileptons at HERA}

In ep interactions, dominated by the $\gamma \gamma$ process.
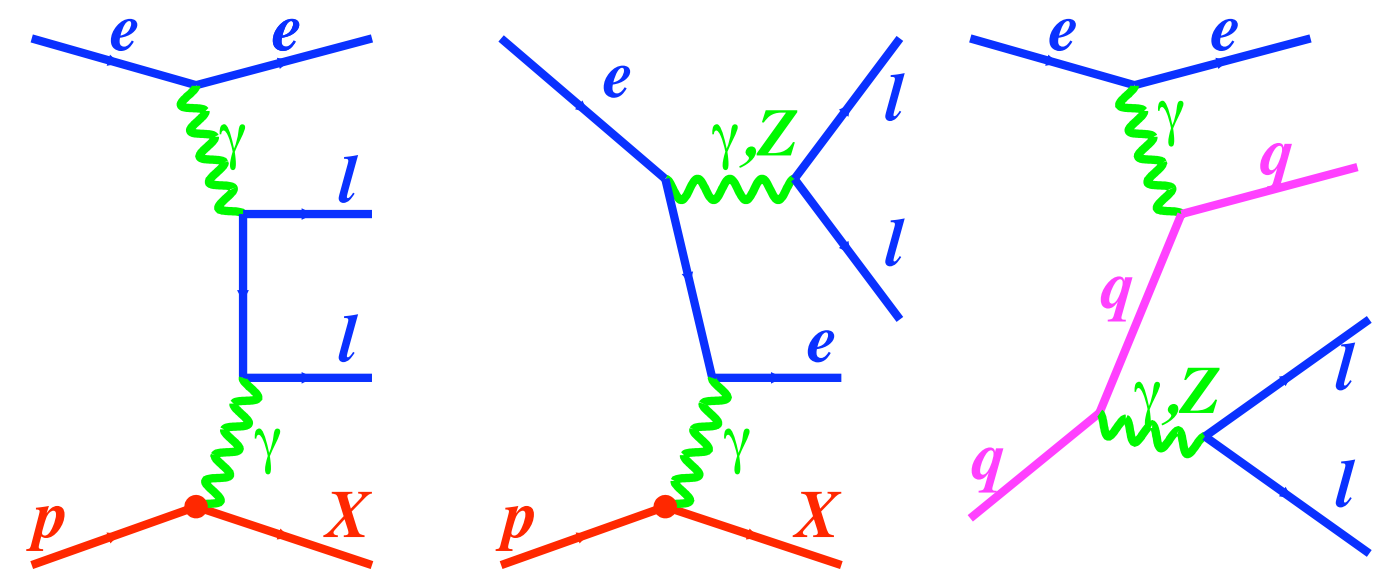

This is a QED process: the predictions from the Standard Model (SM) are very precise.

The SM cross section at high masses, high $\mathrm{p}_{\mathrm{T}}$ is low: we can look for new phenomena.

Background: NC DIS, QED Compton for events with electrons. 


\section{Strategy of the analysis}

- Events are selected by requiring the presence of at least two isolated high- $\mathrm{p}_{\mathrm{T}}$ leptons (electrons or muons) in the final state.

- Depending on the number and the flavours of the leptons, the events are classified into independent samples.

- ee sample: 2 electrons were found, and no other lepton;

- eee: 3 electrons are found, and no other lepton; does not contain the ee sample;

- eu: 1 electron and 1 muon;

- and so on for e $\mu \mu, \mu \mu \ldots$

- Each sample is compared to the SM predictions, looking for possible deviations. The mass of the two highest $\mathrm{p}_{\mathrm{T}}$ leptons, $\mathrm{M}_{12}$, and the $\Sigma \mathrm{p}_{\mathrm{T}}$ of all the leptons are considered. 


\section{Data selection}

\section{Electrons:}

- Identified in $5^{\circ}<\theta<175^{\circ}$, with $\mathrm{E}>5 \mathrm{GeV}$ for $\theta>150^{\circ}, 10 \mathrm{GeV}$ elsewhere $\left(\mathrm{H} 1: 5 \mathrm{GeV}\right.$ up to $\left.20^{\circ}\right)$.

- Isolated (looking at tracks and calorimeter deposits).

Muons:

- Identified in $20^{\circ}<\theta<160^{\circ}$, with $\mathrm{p}_{\mathrm{T}}>2 \mathrm{GeV}$.

- Isolated from tracks.

At least 2 leptons have to be in $20^{\circ}<\theta<150^{\circ}$, with $p_{\mathrm{T}}>10,5 \mathrm{GeV}$.

- Events are assigned to exclusive classes depending on the number and flavour of leptons.

- All possible topologies investigated.

$20^{\circ}$ 


\section{Observed topologies}

ZEUS $\left(\mathcal{L}=480 \mathrm{pb}^{-1}\right)$

\begin{tabular}{|c|c|c||c|c|c|}
\hline Topology & Data & Total SM & Pair Production & NC DIS & Compton \\
\hline$e e$ & 545 & $563_{-37}^{+29}$ & $429_{-29}^{+21}$ & $74 \pm 5$ & $60 \pm 10$ \\
$\mu \mu$ & 93 & $106 \pm 12$ & $106 \pm 12$ & \multicolumn{2}{|c|}{$<0.5$} \\
$e \mu$ & 46 & $42 \pm 4$ & $37_{-4}^{+3}$ & \multicolumn{2}{|c|}{$4.5 \pm 1.2$} \\
$e e e$ & 73 & $75_{-4}^{+5}$ & $73_{-5}^{+4}$ & $<1 \mid<3$ \\
$e \mu \mu$ & 47 & $48 \pm 5$ & $48 \pm 5$ & \multicolumn{2}{|c|}{$<0.5$} \\
$e e e e$ & 1 & $0.9_{-0.1}^{+0.5}$ & $0.6 \pm 0.1$ & $<0.4 \mid<1$ \\
$e e \mu \mu$ & 2 & $0.5_{-0.1}^{+0.3}$ & $0.4 \pm 0.1$ & \multicolumn{2}{|c|}{$<0.5$} \\
\hline All 4-leptons & 3 & $1.4_{-0.2}^{+0.6}$ & $1.0 \pm 0.2$ & \multicolumn{2}{|c|}{$<0.5$} \\
\hline$e e(\gamma \gamma$ sample $)$ & 166 & $185_{-14}^{+8}$ & $183_{-14}^{+8}$ & $1.4 \pm 1.0$ & $1.4 \pm 0.6$ \\
$\mu \mu(\gamma \gamma$ sample $)$ & 72 & $85_{-10}^{+9}$ & $85_{-10}^{+9}$ & $<0.5$ \\
\hline \hline
\end{tabular}

Data are overall well described by the SM including pair production and background. Let's look at the high-mass and high- $\Sigma \mathrm{p}_{\mathrm{T}}$ regions. 


\section{Masses for the different topologies}
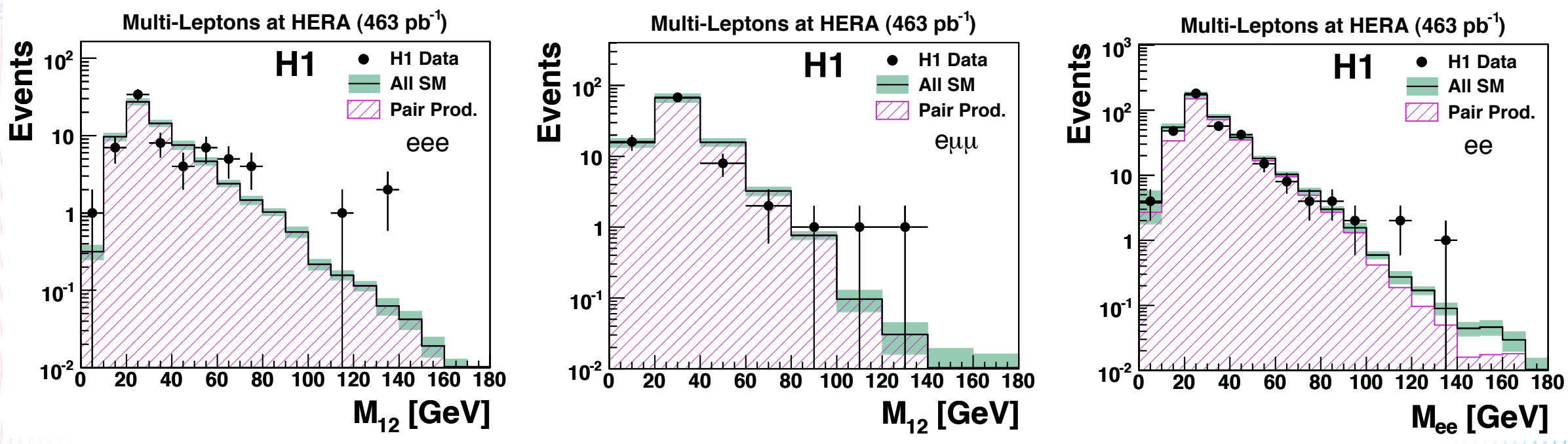

\section{Overall agreement with SM.}

High mass events observed in ee, eee, e $\mu \mu$ topologies.
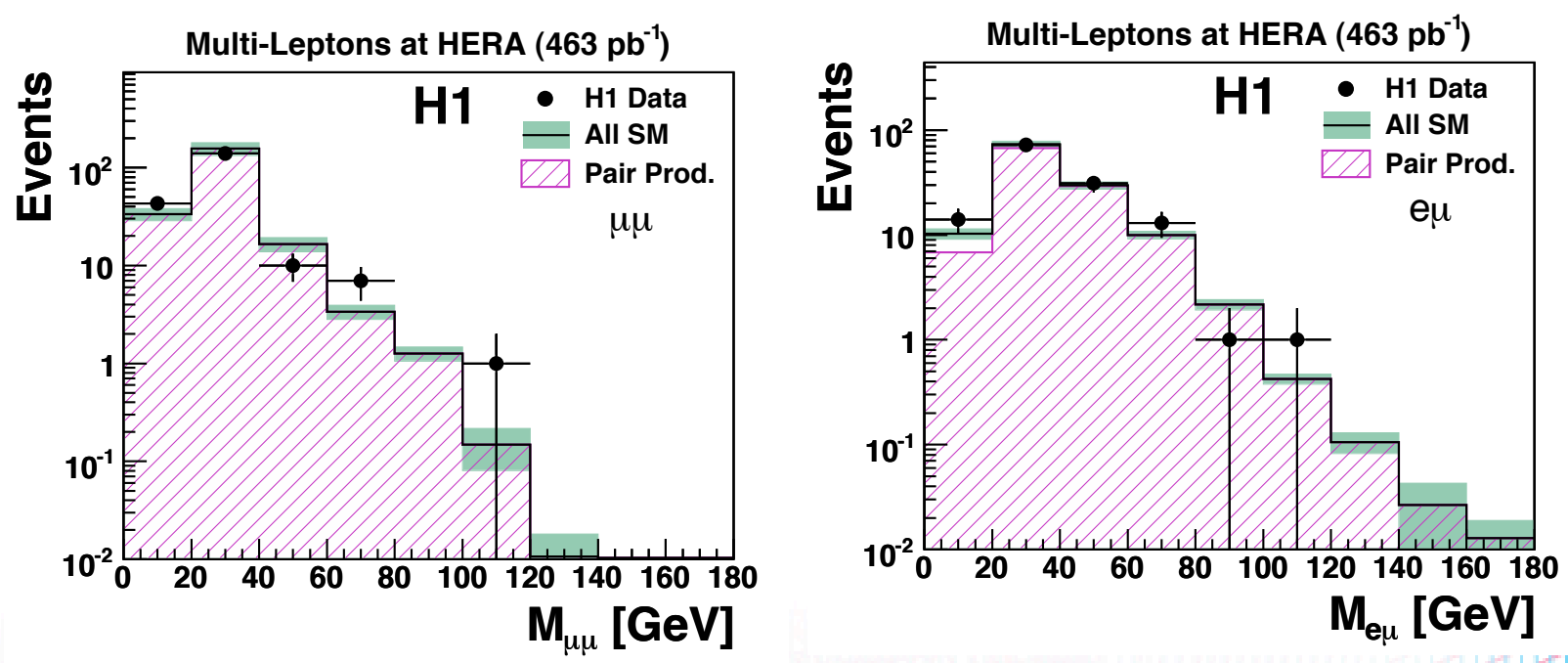


\section{Two H1 events}

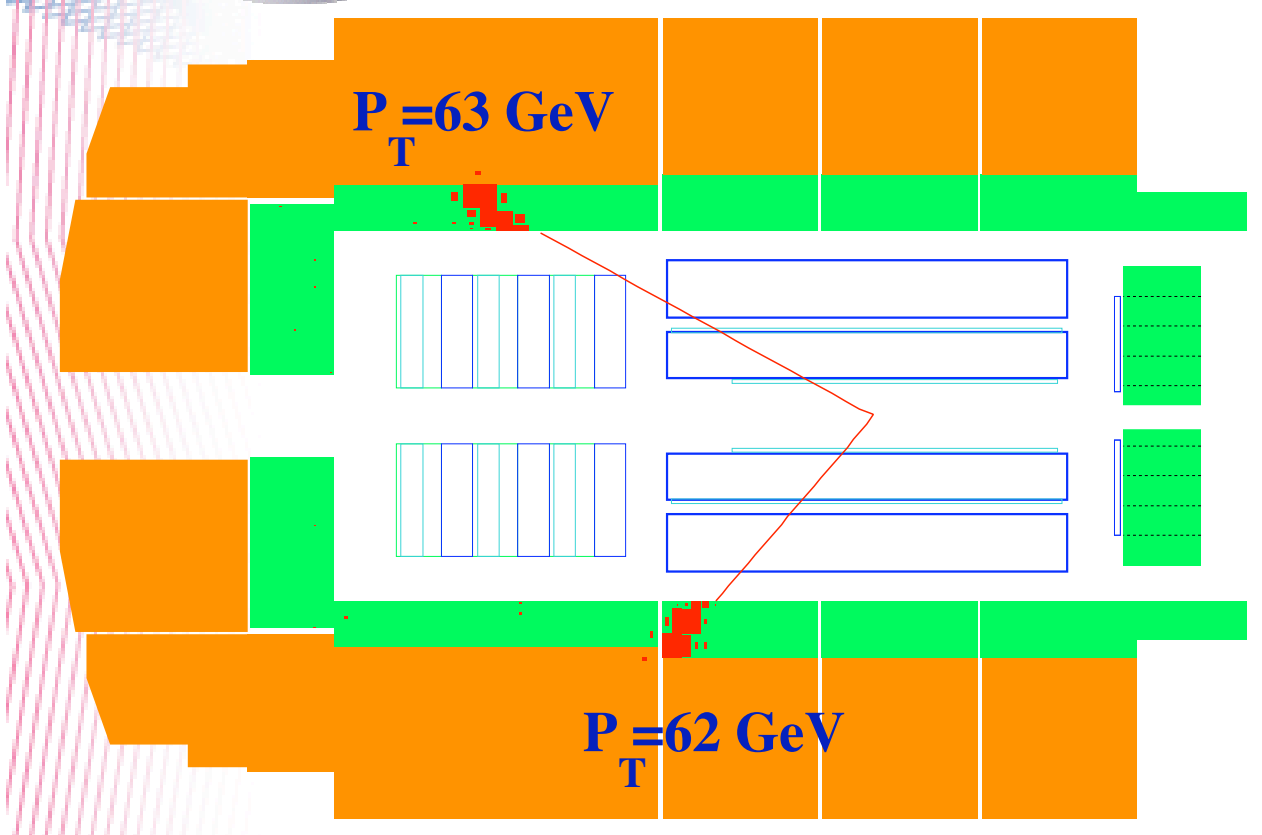

e $\mu \mu$ event: $\mathrm{M}=127 \mathrm{GeV}$, given by the electron and the highest- $\mathrm{p}_{\mathrm{T}}$ muon.

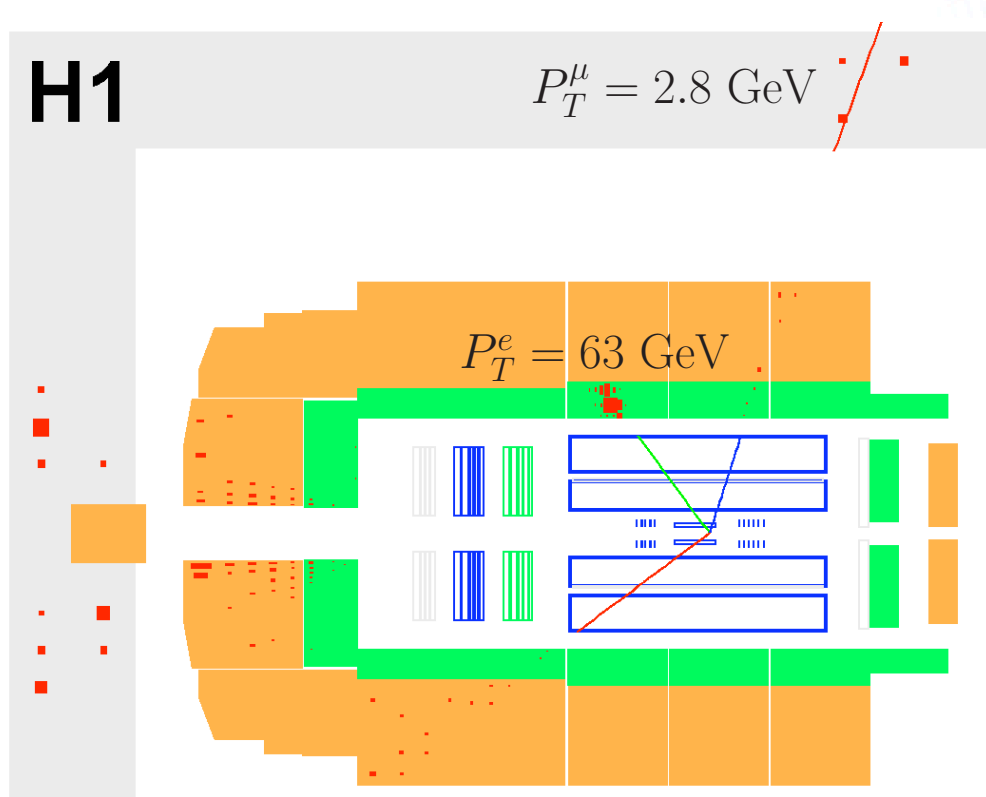

$P_{T}^{\mu}=61 \mathrm{GeV}$ 


\section{$\Sigma p_{\mathrm{T}}$ for the different topologies}

\section{ZEUS}
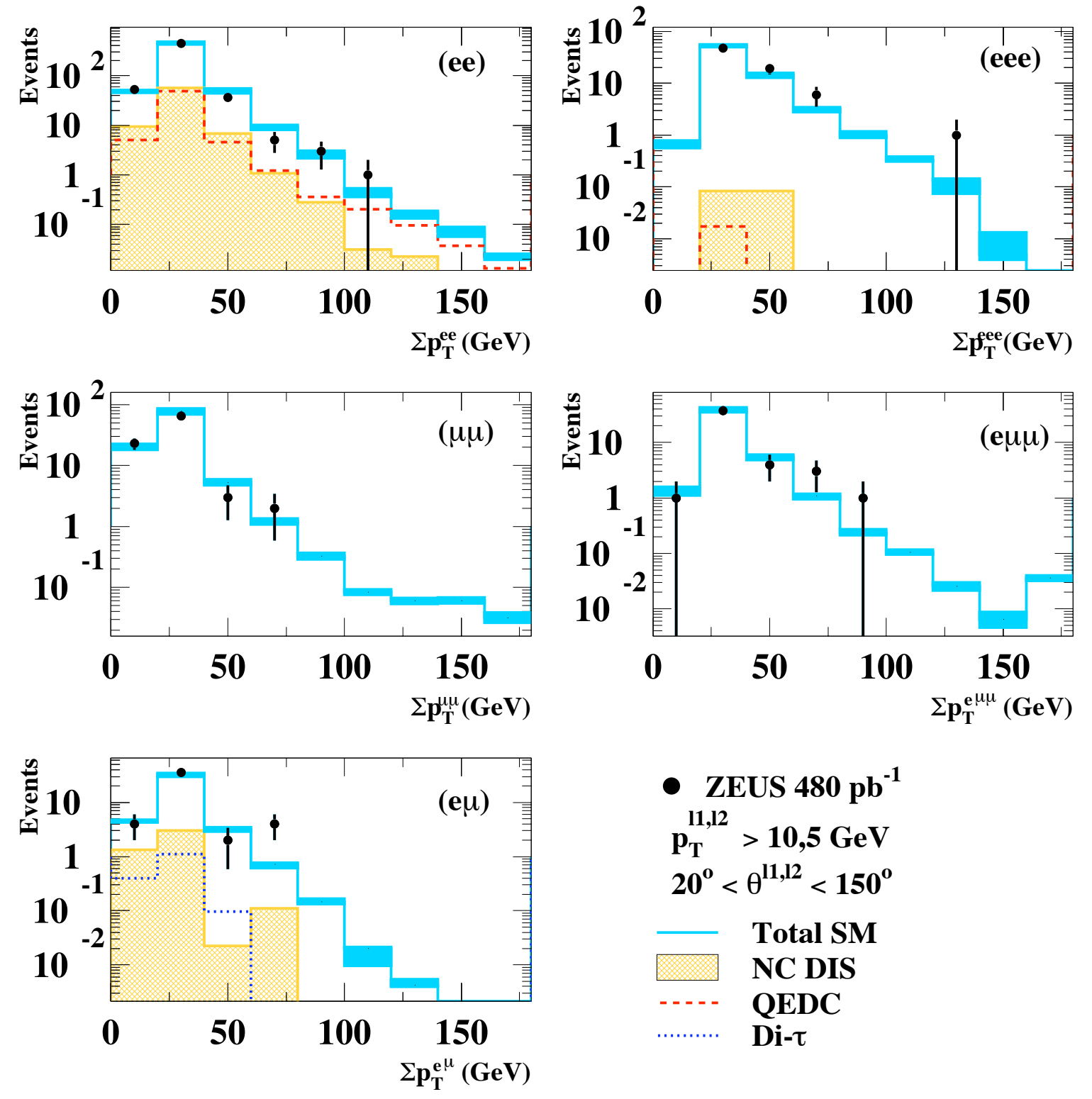

- ZEUS $480 \mathrm{pb}^{-1}$

$\mathrm{p}_{\mathrm{T}}^{\mathrm{I1,12}}>10,5 \mathrm{GeV}$

$20^{\circ}<\theta^{11,12}<150^{\circ}$

Total SM

NC DIS

QEDC

Di- $\tau$
Good agreement with the SM for all the topologies.

2 events observed with high $\Sigma \mathrm{p}_{\mathrm{T}}$. 


\section{Two ZEUS events}

Highest mass event containing only electrons (eee):

$$
\mathrm{m}=113 \mathrm{GeV}
$$

Highest mass event with muons $(\mathrm{e} \mu \mu)$ : $\mathrm{m}=77.5 \mathrm{GeV}$

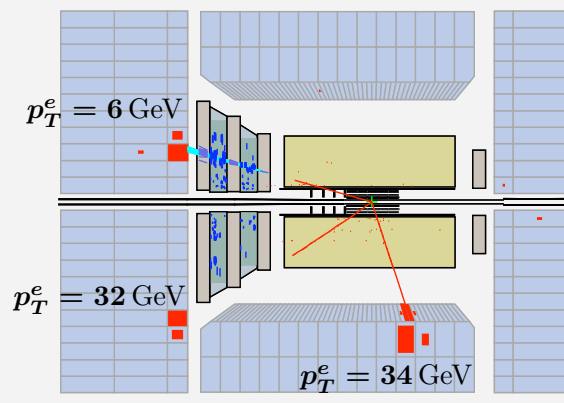

eee

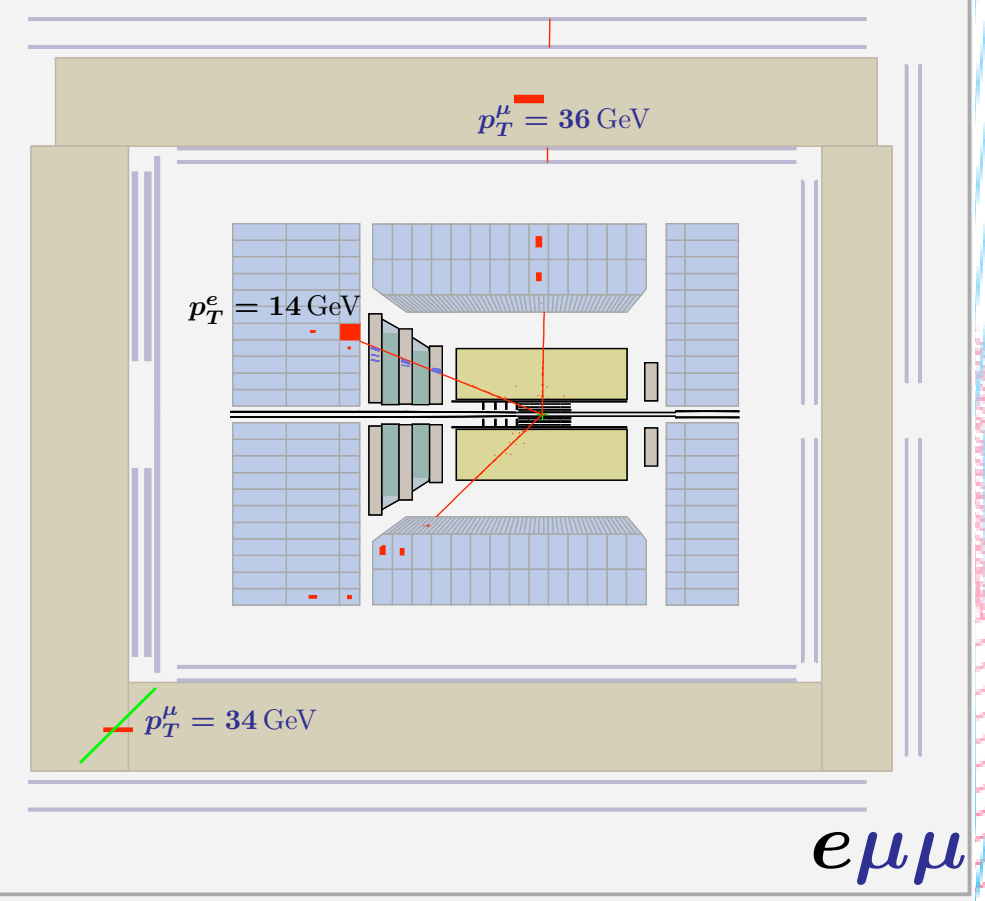




\section{Combination of the topologies}

ZEUS
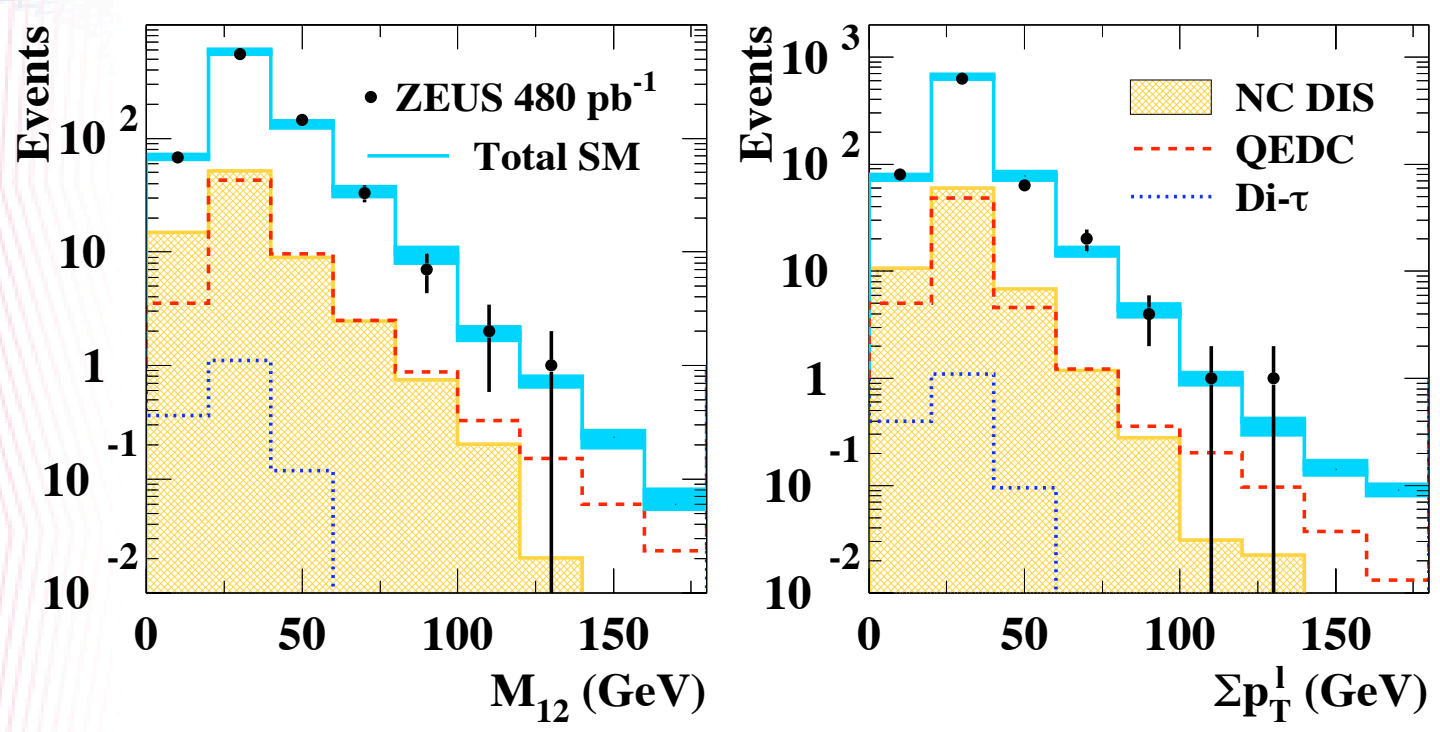

$H 1: 5$ events at high $\Sigma p_{T}$, ZEUS has 2

Let's combine the data...

Good agreement between the data and the SM.

Some events in the high mass and high $\Sigma \mathrm{p}_{\mathrm{T}}$ region.

Multi-Leptons at HERA ( $e^{+} p$ and $e^{-} p, 463 p^{-1}$ )

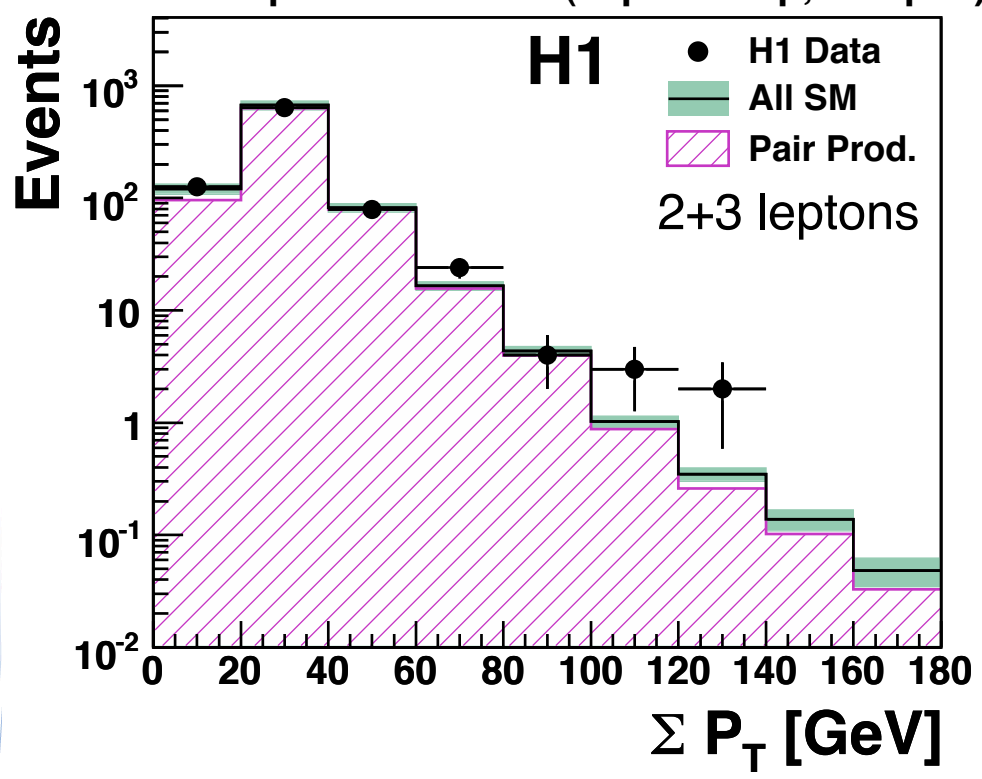




\section{Combined analysis}

- The ZEUS and H1 analyses are done in exactly the same way, apart from:

- $\mathrm{H} 1$ cuts at $5 \mathrm{GeV}$ for the electron in the region $20^{\circ}<\theta<150^{\circ}$ : the cut has been increased to $10 \mathrm{GeV}$ for the combination with ZEUS.

- The measurements are dominated by the statistical error, the systematic uncertainty is uncorrelated between the two experiments (except for the theory error):

- we combine the results with the above assumption, taking the theory uncertainty to be fully correlated (the model is the same). 


\section{Combined topologies table}

Multi-Leptons at HERA $\left(0.94 \mathrm{fb}^{-1}\right)$

\begin{tabular}{|ccccc|}
\hline Selection & Data & SM & Pair Production (GRAPE) & NC DIS + QEDC \\
\hline$e e$ & 873 & $895 \pm 57$ & $724 \pm 41$ & $171 \pm 28$ \\
$\mu \mu$ & 298 & $320 \pm 36$ & $320 \pm 36$ & $<0.5$ \\
$e \mu$ & 173 & $167 \pm 10$ & $152 \pm 9$ & $15 \pm 3$ \\
$e e e$ & 116 & $119 \pm 7$ & $117 \pm 6$ & $<4$ \\
$e \mu \mu$ & 140 & $147 \pm 15$ & $147 \pm 15$ & $<0.5$ \\
\hline$(\gamma \gamma)_{e}$ & 284 & $293 \pm 18$ & $289 \pm 18$ & $4 \pm 1$ \\
$(\gamma \gamma)_{\mu}$ & 235 & $247 \pm 26$ & $247 \pm 26$ & $<0.5$ \\
\hline
\end{tabular}

Good agreement with the Standard Model. Let's look at the high-mass and high- $\Sigma \mathrm{p}_{\mathrm{T}}$ regions. 


\section{Combined mass distributions}
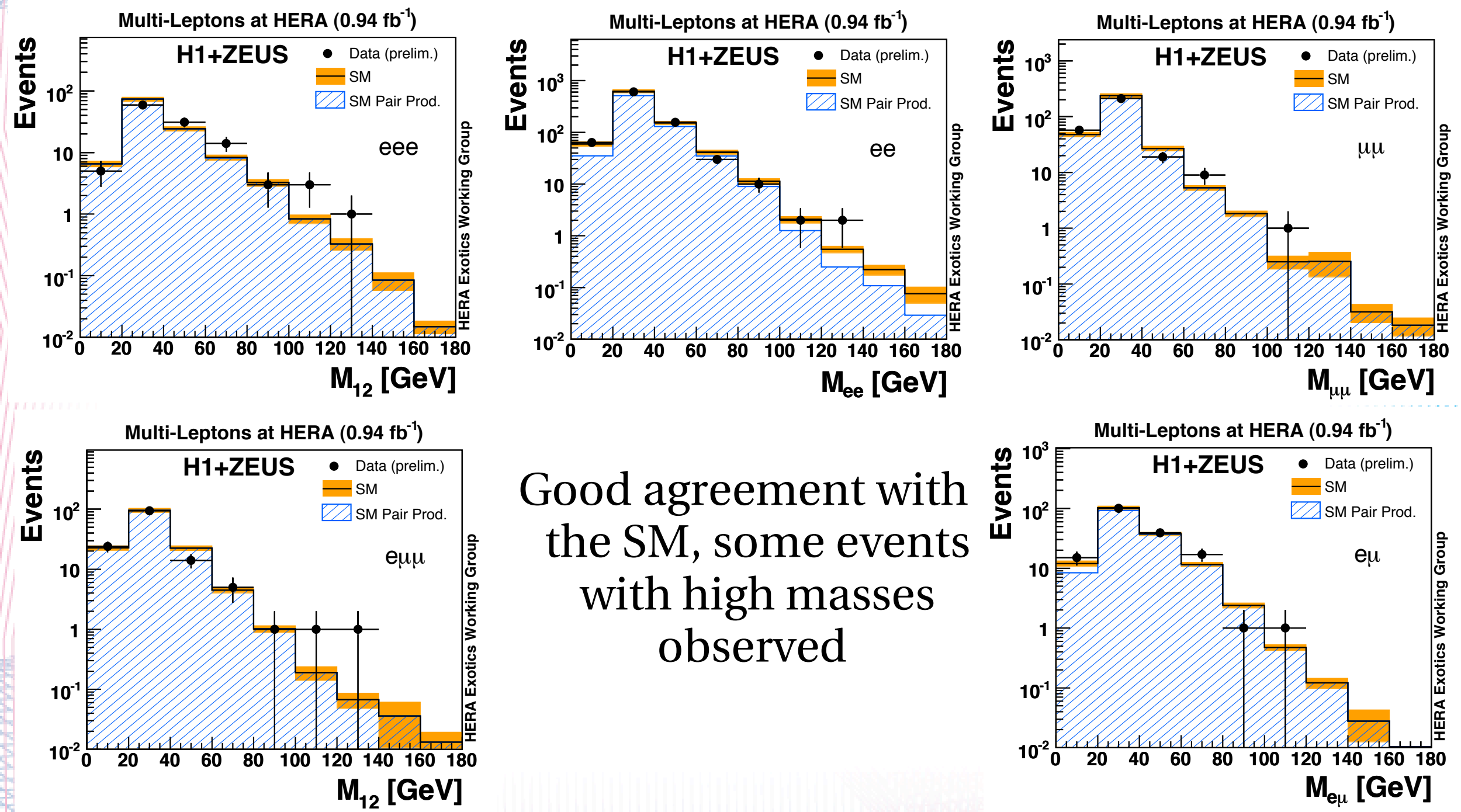


\section{Combined mass table}

\section{All the high-mass events are seen in $\mathrm{e}^{+} \mathrm{p}$ collisions.} 3 events come from
ZEUS, 9 from $\mathrm{H} 1$.
H1+ZEUS Multi-Lepton analysis HERA I+II $\left(0.94 \mathrm{fb}^{-1}\right.$, preliminary $)$

\begin{tabular}{ccccc}
\hline \multicolumn{5}{c}{$M_{12}>100 \mathrm{GeV}$} \\
\hline Selection & Data & SM & Pair Production (GRAPE) & NC DIS + QEDC \\
\hline \multicolumn{5}{c}{ All data $\left(0.94 \mathrm{fb}^{-1}\right)$} \\
\hline$e e$ & 4 & $2.98 \pm 0.28$ & $1.69 \pm 0.15$ & $1.29 \pm 0.16$ \\
$\mu \mu$ & 1 & $0.55 \pm 0.12$ & $0.55 \pm 0.12$ & $<0.01$ \\
$e \mu$ & 1 & $0.65 \pm 0.07$ & $0.64 \pm 0.06$ & $<0.02$ \\
$e e e$ & 4 & $1.27 \pm 0.12$ & $1.27 \pm 0.12$ & $<0.03$ \\
$e \mu \mu$ & 2 & $0.31 \pm 0.06$ & $0.31 \pm 0.06$ & $<0.01$ \\
\hline \multicolumn{5}{c}{$e^{+} p$ collisions $\left(0.56 \mathrm{fb}^{-1}\right)$} \\
\hline$e e$ & 4 & $1.68 \pm 0.18$ & $0.94 \pm 0.11$ & $0.74 \pm 0.12$ \\
$\mu \mu$ & 1 & $0.32 \pm 0.08$ & $0.32 \pm 0.08$ & $<0.01$ \\
$e \mu$ & 1 & $0.40 \pm 0.05$ & $0.39 \pm 0.05$ & $<0.02$ \\
$e e e$ & 4 & $0.79 \pm 0.09$ & $0.79 \pm 0.09$ & $<0.03$ \\
$e \mu \mu$ & 2 & $0.16 \pm 0.04$ & $0.16 \pm 0.04$ & $<0.01$ \\
\hline \multicolumn{5}{c}{$e^{-} p$ collisions $\left(0.38 \mathrm{fb}^{-1}\right)$} \\
\hline$e e$ & 0 & $1.25 \pm 0.13$ & $0.71 \pm 0.11$ & $0.54 \pm 0.08$ \\
$e \mu$ & 0 & $0.23 \pm 0.10$ & $0.23 \pm 0.10$ & $<0.01$ \\
$e e e$ & 0 & $0.26 \pm 0.03$ & $0.25 \pm 0.03$ & $<0.02$ \\
$e \mu \mu$ & 0 & $0.49 \pm 0.74$ & $0.49 \pm 0.07$ & $<0.01$ \\
\hline
\end{tabular}




\section{(116) $\sum \mathbf{p}_{\mathrm{T}}$ distributions}

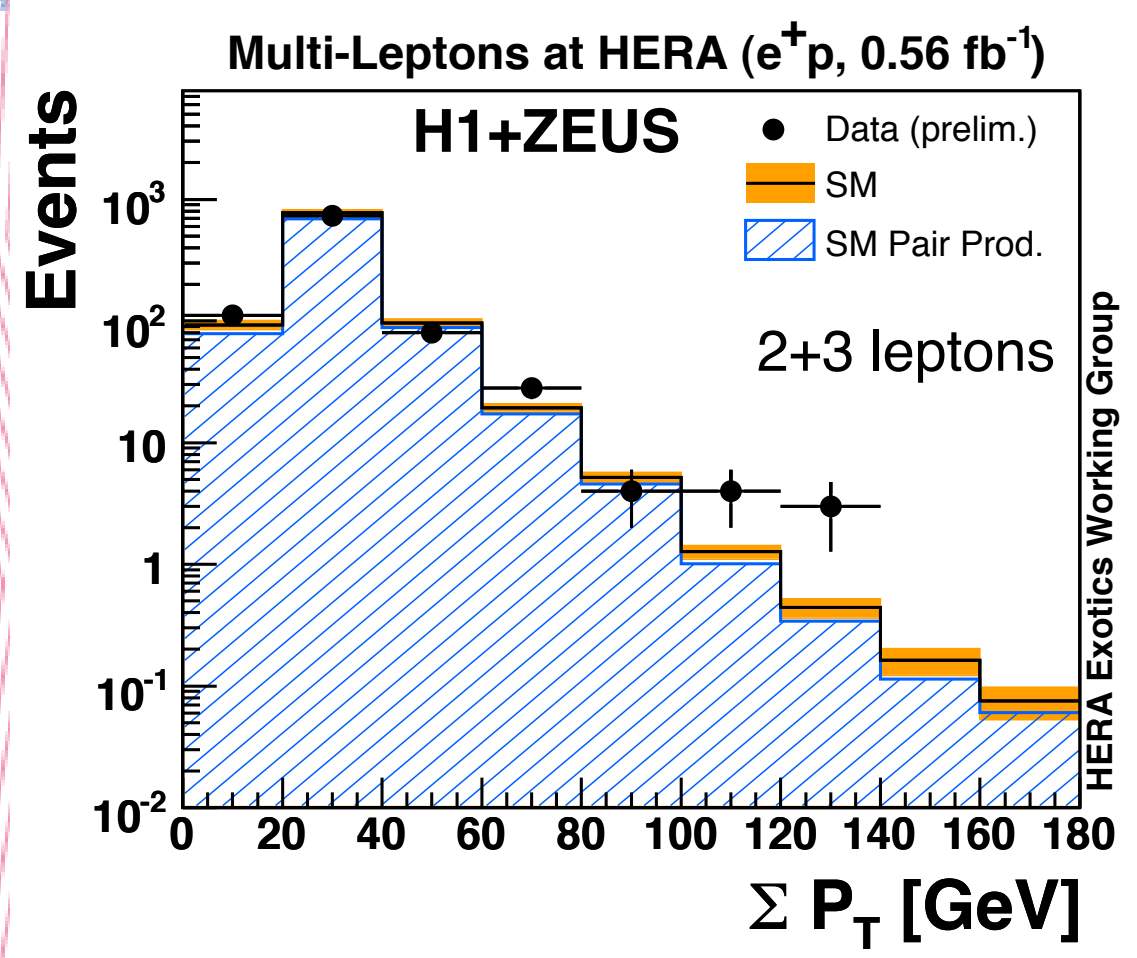

7 high- $\sum \mathrm{p}_{\mathrm{T}}$ events observed in $\mathrm{e}^{+} \mathrm{p}$ data (significance 2.6\%)

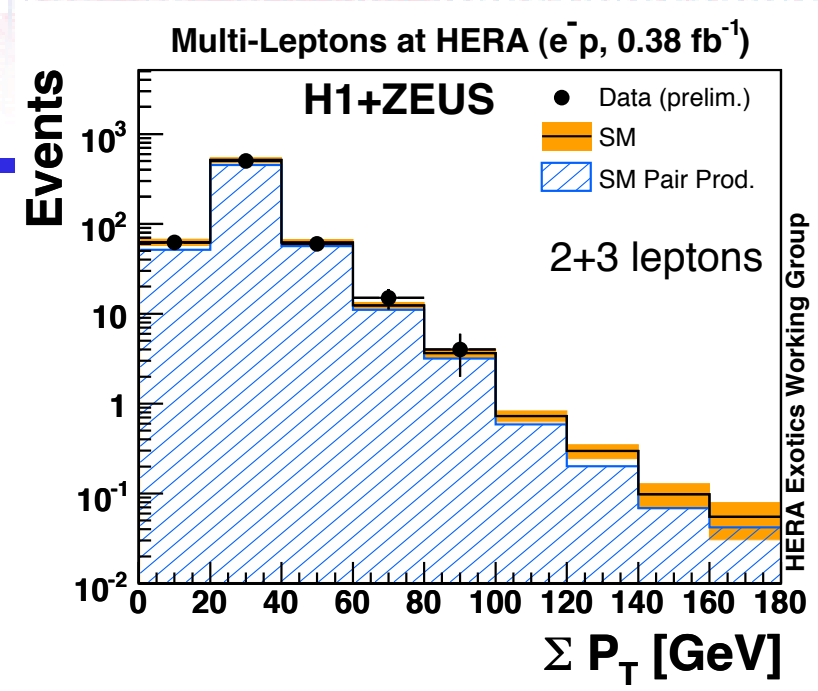

Multi-Leptons at HERA ( $e^{+} p$ and $\left.e^{-} p, 0.94 \mathrm{fb}^{-1}\right)$

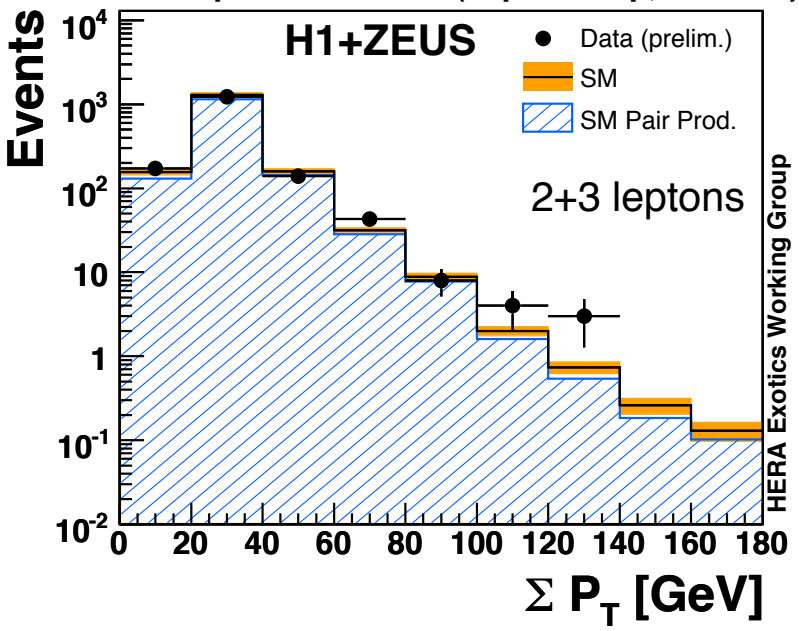

H1+ZEUS Multi-Lepton analysis HERA I+II (0.94 $\mathrm{fb}^{-1}$, preliminary)

\begin{tabular}{ccccc}
\hline Data sample & Data & SM & Pair Production (GRAPE) & NC DIS + QEDC \\
\hline $\mathrm{e}^{+} \mathrm{p}\left(0.56 \mathrm{fb}^{-1}\right)$ & 7 & $1.94 \pm 0.17$ & $1.52 \pm 0.14$ & $0.42 \pm 0.07$ \\
$\mathrm{e}^{-} \mathrm{p}\left(0.38 \mathrm{fb}^{-1}\right)$ & 0 & $1.19 \pm 0.12$ & $0.90 \pm 0.10$ & $0.29 \pm 0.05$ \\
All $\left(0.94 \mathrm{fb}^{-1}\right)$ & 7 & $3.13 \pm 0.26$ & $2.42 \pm 0.21$ & $0.71 \pm 0.10$ \\
\hline
\end{tabular}

iti Monica Turcato

$2.42 \pm 0.21$

$0.71 \pm 0.10$ 


\section{Measurement of the cross sections}

- In order to select a sample enriched in photoproduction events, the cut $E-p_{z}<45 \mathrm{GeV}$ was imposed.

- In this way the sample is constituted by events in which two leptons of the same flavour are found in the final state.

- Cross sections are evaluated for the $\gamma \rightarrow \rightarrow l$ process in the kinematic region:

- leptons must be isolated $(\Delta \mathrm{r}>0.5$ in the pseudorapidity-azimuth plane)

- $\mathrm{Q}^{2}<1 \mathrm{GeV}^{2}, \mathrm{y}<0.82$ (photoproduction regime)

- $\mathrm{p}_{\mathrm{T}}{ }^{11,12}>10,5 \mathrm{GeV}$

- $20^{\circ}<\theta<150^{\circ}$ 


\section{Combined cross sections}

Differential cross sections measured as a function of the mass of the dilepton system and of the $\mathrm{p}_{\mathrm{T}}$ of the highest- $\mathrm{p}_{\mathrm{T}}$ lepton.

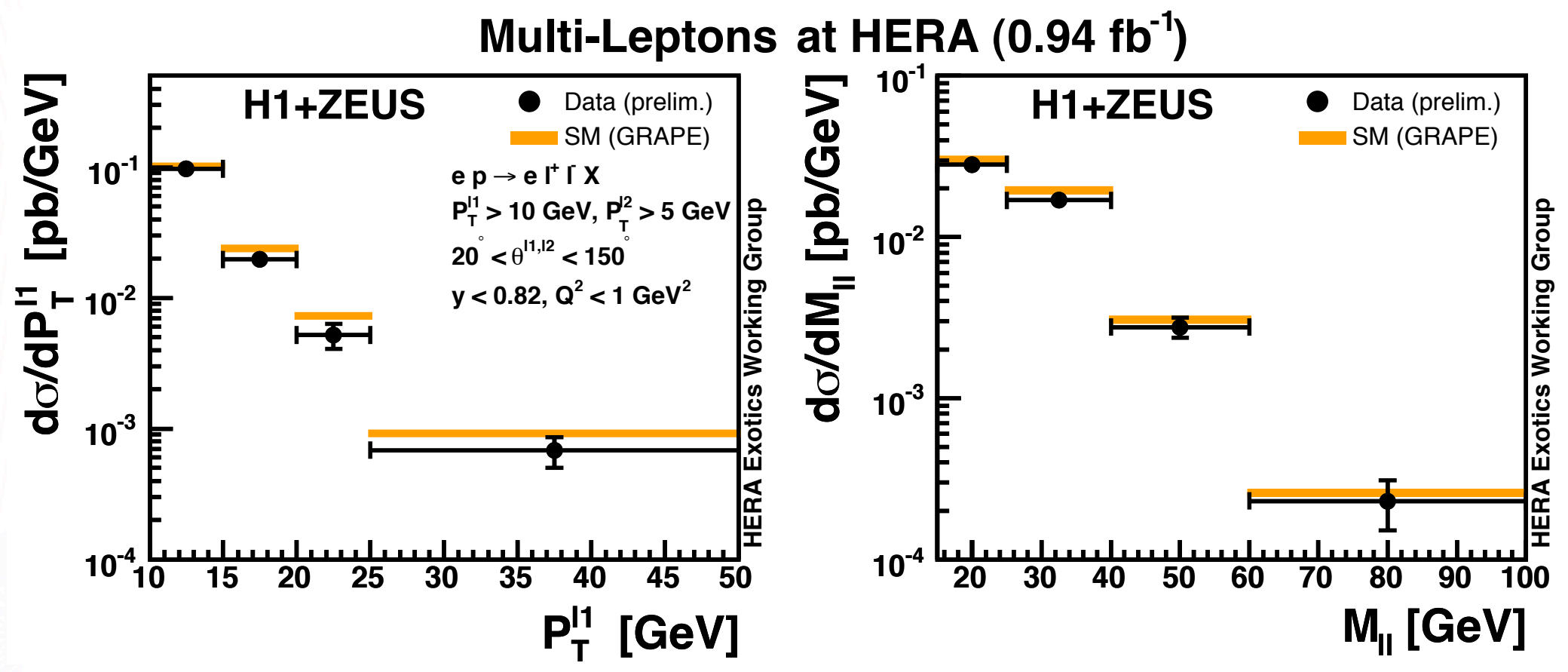




\section{Conclusions}

- Multilepton production has been studied at HERA, looking for possible deviations from the SM in the high mass and high$\sum \mathrm{p}_{\mathrm{T}}$ regions.

- All the event topologies containing electrons and/or muons have been investigated. An overall good agreement with the SM has been found.

- The results of the H1 and ZEUS experiments have been combined to reach best sensitivity: some events with high- $\sum \mathrm{p}_{\mathrm{T}}$ and high masses have been observed, for both experiments only in $\mathrm{e}^{+} \mathrm{p}$ collisions.

- Cross sections for the process $\gamma \gamma \rightarrow l^{+} l^{-}$have been measured using the full available HERA statistics. 


\section{Backup}


ZEUS ditau events HERA II data $\left(\mathrm{L}=\mathbf{0 . 3 6} \mathrm{fb}^{-1}\right)$

\begin{tabular}{|c||c||c|c|c|c}
\hline Topology & All & jet-jet & $e$-jet-jet & $e$-jet & $e$-e-jet \\
\hline D cut & & 0.80 & 0.50 & 0.90 & 0.90 \\
\hline \hline Data & 21 & 14 & 3 & 4 & 0 \\
\hline Total SM & $27.2_{-6.3}^{+7.1}$ & $20.2_{-5.7}^{+6.8}$ & $1.4_{-0.2}^{+3.3}$ & $4.9_{-1.3}^{+3.1}$ & $0.7_{-0.1}^{+4.4}$ \\
ditau MC & $13.2_{-1.0}^{+0.6}$ & $9.1_{-0.8}^{+0.4}$ & $1.4 \pm 0.1$ & $2.2 \pm 0.1$ & $0.5 \pm 0.1$ \\
(purity) & $(49 \%)$ & $(45 \%)$ & $(97 \%)$ & $(46 \%)$ & $(74 \%)$ \\
\hline
\end{tabular}

\section{Di- $\tau$}

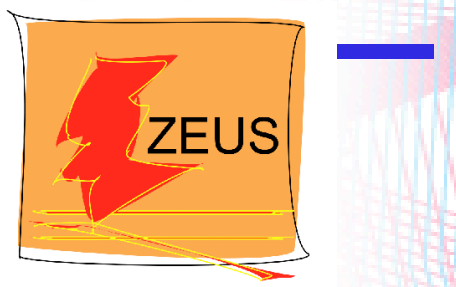

\section{ZEUS}

Analysis performed on the HERAII data.

Topologies with jets and electrons investigated.



\section{Masses for the different topologies}

\section{ZEUS}
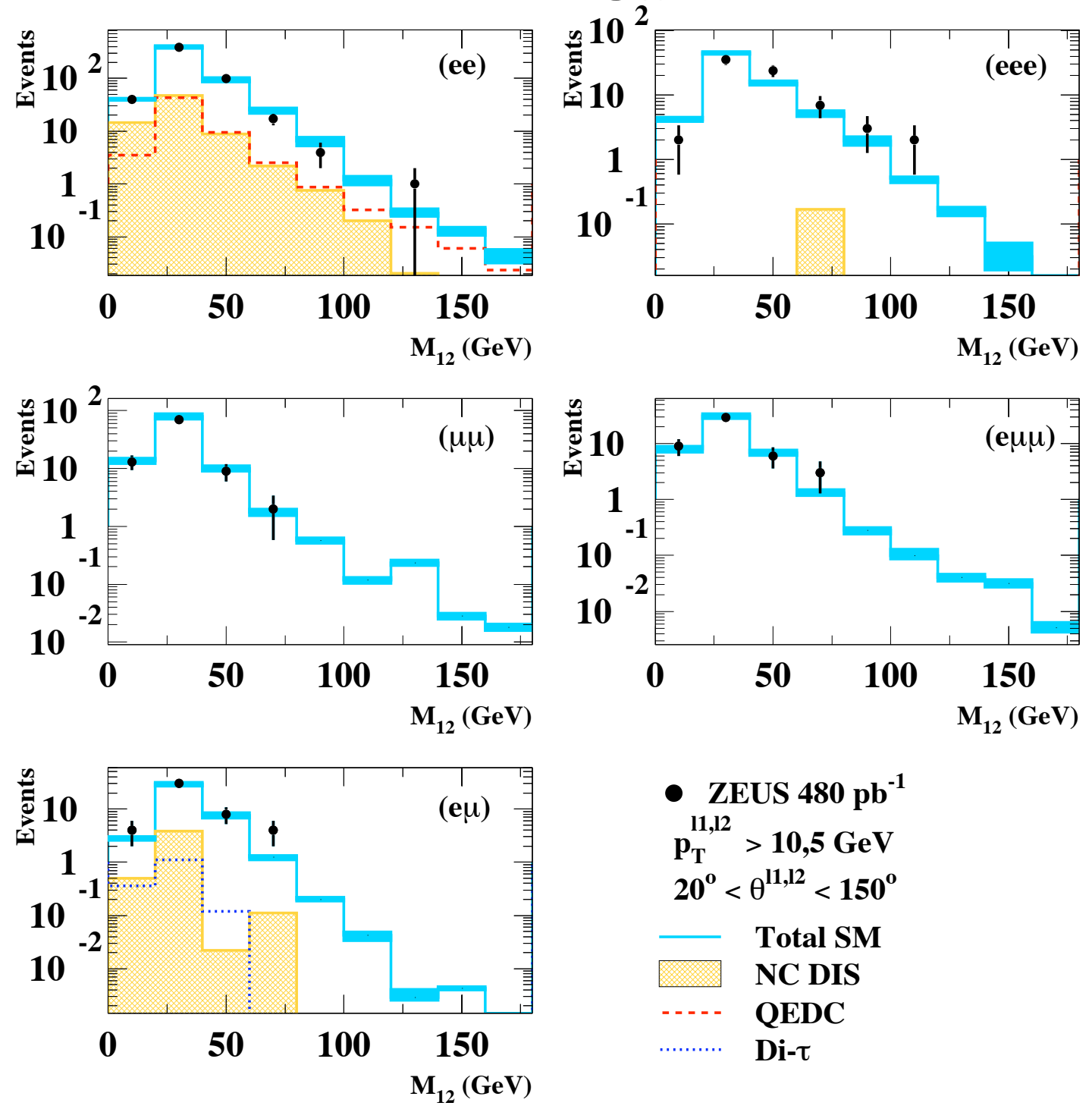

- ZEUS $480 \mathrm{pb}^{-1}$ $\mathbf{p}_{\mathrm{T}}^{\mathrm{I1,12}}>10,5 \mathrm{GeV}$ $20^{\circ}<\theta^{11,12}<150^{\circ}$

Total SM NC DIS QEDC $\mathrm{M}_{12}(\mathrm{GeV})$ 